\title{
Considerations on bubble fragmentation models
}

\author{
C. MARTÍNEZ-BAZÁN , J. RODRÍGUEZ-R ODRÍGUEZ, \\ G. B. DEANE, J. L. MONT AÑES AND J. C. LASHERAS \\ Área de Mecánica de Fluidos. Departamento de Ingenieria Mecánica y Minera. Universidad Jaén, \\ Campus de las Lagunillas, 23071 Jaén, Spain \\ Área de Mecánica de Fluidos, Departamento de Ingenieria Térnica y de Fluidos. \\ Universidad Carlos III de Madrid, 28911 Leganés, Spain \\ Marine Physical Laboratory. Scripps Institution of Oceanography. La Jolla. CA 92093-0238. USA \\ Departamento de Motopropulsión y Temofluidodinámica. Universidad Politécnica de Madrid, \\ Plaza del Cardenal Cisneros 3. 28040 Madrid. Spain
}

Department of Mechanical and Aerospace Engineering, University of California San Diego. La Jolla, CA 92093-0411, USA

In this paper we describe the restrictions that the probability density function (p.d.f.) of the size of particles resulting from the rupture of a drop or bubble must satisfy. Using conservation of volume, we show that when a particle of diameter, $D_{0}$, breaks into exactly two fragments of sizes $D$ and $D_{2}=\left(D_{0}^{3}-D^{3}\right)^{1 / 3}$ respectively, the resulting p.d.f., $f\left(D ; D_{0}\right)$. must satisfy a symmetry relation given by $D_{2}^{2} f\left(D ; D_{0}\right)=D^{2} f\left(D_{2} ; D_{0}\right)$, which does not depend on the nature of the underlying fragmentation process. In general, for an arbitrary number of resulting particles, $m\left(D_{0}\right)$, we determine that the daughter p.d.f. should satisfy the conservation of volume condition given by $m\left(D_{0}\right) \int_{0}^{D_{0}}\left(D / D_{0}\right)^{3} f\left(D ; D_{0}\right) \mathrm{d} D=1$. A detailed analysis of some contemporary fragmentation models shows that they may not exhibit the required conservation of volume condition if they are not adequately formulated. Furthermore, we also analyse several models proposed in the literature for the breakup frequency of drops or bubbles based on different principles, $g\left(\varepsilon, D_{0}\right)$. Although, most of the models are formulated in terms of the particle size $D_{0}$ and the dissipation rate of turbulent kinetic energy, $\varepsilon$, and apparently provide different results, we show here that they are nearly identical when expressed in dimensionless form in terms of the Weber number, $g^{*}\left(W e_{t}\right)=g\left(\varepsilon, D_{0}\right) D_{0}^{2 / 3} \varepsilon^{-1 / 3}$, with $W e_{t} \sim \rho \varepsilon^{2 / 3} D_{0}^{5 / 3} / \sigma$, where $\rho$ is the density of the continuous phase and $\sigma$ the surface tension.

Key words: breakup/coalescence

\section{Introduction}

The fragmentation of drops and bubbles in sheared or turbulent flows has a number of important applications in engineering and earth science fields. Bubble fragmentation in whitecaps, for example, plays an important role in the size distribution of bubbles created by breaking waves, which contributes to air-sea gas flux, aerosol production, ocean albedo, wave breaking energetics and the generation of underwater ambient noise (Melville 1996; Deane \& Stokes 2002). The general principles controlling 
the breakup of drops and bubbles in turbulent flows have been established since the works of Kolmogorov (1949) and Hinze (1955) in the early 1950s, but an exact mathematical description of this fundamental fluid dynamical process has not yet been formulated.

Since the seminal works of Kolmogorov (Kolmogorov 1949) and Hinze (Hinze 1955), a number of models for bubble fragmentation have been presented, including the phenomenological fragmentation model of Martínez-Bazán, Montañes \& Lasheras $(1999 a, b)$ that describes the breakup frequency, $g\left(D_{0}\right)$, and probability density function (p.d.f.) of daughter bubbles, $f\left(D ; D_{0}\right)$, produced by the fragmentation of air bubbles in homogeneous, isotropic turbulence (see Lasheras et al. 2002, for a recent review of bubble fragmentation models).

The implementation of some breakup models in the population balance equation may lead to the impression that they do not conserve volume since some of the equations for the bubble-size p.d.f. published in the literature are expressed in terms of bubble diameter instead of volume. Thus, some of the turbulent breakup models are based on phenomenological hypotheses and, although they have been developed under the original principle of conservation of volume, especially the binary models, for convenience they are expressed in terms of the bubble diameter and do not always respect the volume-conservation condition. This issue has been also pointed out by Zaccone et al. (2007), who developed an empirical approach to determine the breakup mechanisms in stirred dispersions and an appropriate physical model for the daughter-drop p.d.f, and reported that many models, with the exception of Coulaloglou \& Tavlarides (1977) and Luo \& Svensen (1996), do not always preserve volume. In fact, they emphasized that models that do not conserve volume lead to an unphysical evolution of the predicted volume fraction.

In this paper, in $\$ 2$, we state a simple relation that any volume-conserving fragmentation p.d.f. must satisfy, irrespective of the underlying fragmentation physics, making it a simple matter to determine if a model is volume conserving or not. In particular, in $\$ 3$, we review the models described in Lasheras et al. (2002) and establish whether they satisfy the conservation of volume and symmetry conditions, and show that the binary models are volume conserving when the equations for the p.d.f.s are expressed in terms of volume rather than diameter. In addition, we also propose a modification of Martínez-Bazán et al. (1999b) fragmentation model that satisfies conservation of volume and incorporates slightly different physical ideas to include the effect of surface energy of the resulting bubbles. In $\$ 4$ we review some of the breakup frequency models to show that most of them provide nearly identical functions when they are conveniently expressed in dimensionless form in terms of the turbulent Weber number. Finally, in $\$ 5$ we integrate the population balance equation to determine the evolution of the distribution of bubbles in a turbulent water jet using different models for the daughter-bubble p.d.f. and compare them with the experimental results of Martinez-Bazán et al. (1999b), and $\$ 6$ is devoted to conclusions.

\section{Mathematical constraints to the daughter-bubble-size p.d.f.}

This section establishes the constraints that any breakup model for the daughterbubble p.d.f. must satisfy. Such conditions can be easily extracted, for any given number of fragments generated, as follows.

The time rate of change of the number density of bubbles (or drops) with respect to position and time of a certain size $D$ existing in a time $t$, at a given position $\boldsymbol{x}$, 
$n(D, x, t)$, can be described by the following Boltzmann-type equation, commonly named the population balance equation (Williams 1985):

$$
\frac{\partial n}{\partial t}+\nabla \cdot(v n)=-\frac{\partial}{\partial D}(R n)+\dot{Q}_{b}+\dot{Q}_{c},
$$

where $v(D, x, t)$ is the mean velocity of all the bubbles of size $D, R=\mathrm{d} D / \mathrm{d} t$ is the rate of change of the bubbles of size $D$ due to dissolution and, $\dot{Q}_{b}(D, x, t)$ and $\dot{Q}_{c}(D, x, t)$ are the rate of change of $n(D, x, t)$ due to breakup and coalescence respectively. For very dilute systems, where $\dot{Q}_{c}=0$, and in the absence of dissolution effects, the rate of change of the number density of bubbles can simply be written as the sum of the 'death' rate of bubbles of size $D$ due to their breakup into smaller ones, and the 'birth' rate of particles of size $D$ resulting from the breakup of larger ones:

$$
\frac{\partial n(D)}{\partial t}+\nabla \cdot[v n(D)]=\int_{D}^{\infty} m\left(D_{0}\right) f\left(D ; D_{0}\right) g\left(D_{0}\right) m\left(D_{0}\right) \mathrm{d} D_{0}-g(D) n(D),
$$

where $m\left(D_{0}\right)$ is the mean number of daughter bubbles formed upon the breakup of a mother bubble of size $D_{0}, f\left(D ; D_{0}\right)$ is the size p.d.f. of daughter bubbles generated from the rupture of a mother bubble of size $D_{0}$ and $g(D)$ is the breakup frequency of bubbles of size $D$. In (2.2), although $n, v, m, f$ and $g$ are in general functions of $D, x$ and $t$, only the dependence of $D$ has been indicated for convenience. It should be noticed here that $f\left(D ; D_{0}\right)$ represents a p.d.f. with regard to $D$, parametrized by $D_{0}$, and must not be understood as a joint p.d.f. describing the distribution of both $D$ and $D_{0}$. Thus, $f\left(D ; D_{0}\right)$ must satisfy the normalization condition of a p.d.f., $\int_{0}^{\infty} p . d . f .(x) \mathrm{d} x=1$, that since $f\left(D ; D_{0}\right)=0$ for $D>D_{0}$, can be expressed as $\int_{0}^{D_{0}} f\left(D ; D_{0}\right) \mathrm{d} D=1$.

If we consider a cloud of bubbles immersed in a homogeneous and isotropic turbulent flow moving with the fluid, the population balance equation (2.2) may be written as

$$
\frac{\mathrm{D} n(D)}{\mathrm{D} t}=\int_{D}^{\infty} m\left(D_{0}\right) f\left(D ; D_{0}\right) g\left(D_{0}\right) n\left(D_{0}\right) \mathrm{d} D_{0}-g(D) n(D) .
$$

where $\mathrm{D} n(D) / \mathrm{D} t=\partial n(D) / \partial t+\boldsymbol{u} \cdot \nabla[n(D)], \boldsymbol{u}(\boldsymbol{x}, t)$ is the fluid velocity and, although not explicitly expressed in (2.3) for simplicity, $f\left(x, t, D ; D_{0}\right), m(x, t, D)$ and $g(x, t, D)$ depend on the local turbulent properties of the flow at a given position $x$ in a time $t$. Multiplying (2.3) by $D^{3}$, one obtains

$$
\frac{\mathrm{D}\left[D^{3} n(D)\right]}{\mathrm{D} t}=\int_{D}^{\infty} m\left(D_{0}\right) f\left(D ; D_{0}\right)\left(\frac{D}{D_{0}}\right)^{3} g\left(D_{0}\right) D_{0}^{3} n\left(D_{0}\right) \mathrm{d} D_{0}-g(D) D^{3} n(D),
$$

where $D^{3} n(D)$ represents the volume of particles of a given size $D$. Integrating (2.4) from $D=0$ to $D=\infty$, using the fact that $f\left(D ; D_{0}\right)$ is zero for $D>D_{0}$, and inverting the order of integration into the double integral, yields

$$
\int_{0}^{\infty} g(D) D^{3} n(D) \mathrm{d} D=\int_{0}^{\infty} m\left(D_{0}\right)\left[\int_{0}^{D_{0}}\left(\frac{D}{D_{0}}\right)^{3} f\left(D ; D_{0}\right) \mathrm{d} D\right] g\left(D_{0}\right) D_{0}^{3} n\left(D_{0}\right) \mathrm{d} D_{0} .
$$

Notice that the left-hand side of (2.4) has been cancelled in (2.5) because the total volume of bubbles is preserved. Finally, since the integration variable in the left-hand side of (2.5) may be changed from $D$ to $D_{0}$, and the resulting equality must be valid 
for any $n\left(D_{0}\right) g\left(D_{0}\right)$, one obtains

$$
m\left(D_{0}\right) \int_{0}^{D_{0}}\left(\frac{D}{D_{0}}\right)^{3} f\left(D ; D_{0}\right) \mathrm{d} D=1 .
$$

which is the volume-conserving condition for an arbitrary number of fragments, $m\left(D_{0}\right)$. The above condition, together with the normalization condition of the p.d.f. $f\left(D ; D_{0}\right)$.

$$
\int_{0}^{D_{0}} f\left(D ; D_{0}\right) \mathrm{d} D=1
$$

are the conditions that any model for the daughter-bubble p.d.f. must satisfy. Notice that, in the case of binary breakup, imposing volume conservation is equivalent to satisfying the following symmetry relation:

$$
f_{V}\left(V ; V_{0}\right)=f_{V}\left(V_{2} ; V_{0}\right) \text {, }
$$

with $V_{2}=V_{0}-V$, that establishes that the probability of formation of a bubble of volume $V$ must be equal to the probability of formation of its complementary bubble of volume $V_{2}=V_{0}-V$. Furthermore, since $f_{V}\left(V ; V_{0}\right) \mathrm{d} V=f\left(D ; D_{0}\right) \mathrm{d} D$ with $\mathrm{d} V=\pi / 2 D^{2} \mathrm{~d} D,(2.8)$ can be expressed in terms of the bubble diameter as

$$
D_{2}^{2} f\left(D ; D_{0}\right)=D^{2} f\left(D_{2} ; D_{0}\right),
$$

where $D_{2}=\left(D_{0}^{3}-D^{3}\right)^{1 / 3}$ is the diameter of the complementary bubble. Equations (2.6)-(2.7) are equivalent to the constraints on the zeroth and first moments of the daughter distribution function established by Diemer \& Olson (2002).

\section{Review of previous models for the daughter-bubble p.d.f.}

\subsection{Martinez-Bazán et al. (1999 b) model}

Martínez-Bazán et al. (1999b) proposed a phenomenological model to determine the probability of formation of a pair of bubbles of sizes $D$ and $\left(D_{0}^{3}-D^{3}\right)^{1 / 3}$ generated upon the turbulent breakup of a mother bubble of characteristic size $D_{0}$ given by

$$
\left.\begin{array}{lll}
P\left(D^{*}\right)=0 & \text { for } & D^{*}<D_{\min ,}^{*} \\
P\left(D^{*}\right) \propto\left[\Delta \tau_{11}\right]\left[\Delta \tau_{t 2}\right] & \text { for } & D_{\min }^{*} \leqslant D^{*} \leqslant D_{\max }^{*} \\
P\left(D^{*}\right)=0 & \text { for } & D^{*}>D_{\max }^{*}
\end{array}\right\}
$$

with $D^{*}=D / D_{0}, D_{\min }^{*}=D_{\min } / D_{0}=[12 \sigma /(\beta \rho)]^{3 / 2} D_{0}^{-5 / 2} \varepsilon^{-1}$ and $D_{\max }^{*}=\left(1-D_{\min }^{* 3}\right)^{1 / 3}$. The above equation indicates that the probability of formation of a certain pair of bubbles should be weighted by the product of the excess stresses associated with the length scales corresponding to each bubble size, $\Delta \tau_{f 1}=1 / 2 \rho \beta(\varepsilon D)^{2 / 3}-$ $6 \sigma / D_{0}$ and $\Delta \tau_{t 2}=1 / 2 \rho \beta\left(\varepsilon D_{2}\right)^{2 / 3}-6 \sigma / D_{0}$, with $D_{2}=D_{0}\left[1-\left(D / D_{0}\right)^{3}\right]^{1 / 3}$ provided by conservation of volume. Here $\beta=8.2, \sigma$ is the surface tension, $\rho$ the liquid density and $\varepsilon$ the dissipation rate of turbulent kinetic energy.

Equation (3.1) can be expressed as

$$
P\left(D^{*}\right) \propto\left(\frac{1}{2} \rho \beta\left(\varepsilon D_{0}\right)^{2 / 3}\right)^{2}\left[D^{* 2 / 3}-A^{5 / 3}\right]\left[\left(1-D^{* 3}\right)^{2 / 9}-A^{5 / 3}\right],
$$

in the range $D_{\min }^{*} \leqslant D^{*} \leqslant D_{\max }^{*}$, where $\Lambda=D_{c} / D_{0}$ and $D_{c}=[12 \sigma /(\beta \rho)]^{3 / 5} \varepsilon^{-2 / 5}$ simply represents the critical diameter of a bubble such that the turbulent stresses acting on its surface, $\tau_{t}=1 / 2 \rho \beta\left(\varepsilon D_{c}\right)^{2 / 3}$, are equal to the confining stresses due to surface tension, $\tau_{s}=6 \sigma / D_{c}$. In other words, $D_{c}$ is the diameter of the largest bubble which will not break due to the turbulent action of the flow. Notice that, in (3.2), the 
parameter $A^{5 / 3}$ can also be interpreted as the inverse of the mother-bubble Weber number, defined as $W e_{t}=\rho \beta \varepsilon^{2 / 3} D_{0}^{5 / 3} / 12 \sigma$, providing

$$
P\left(D^{*}\right) \propto\left(\frac{1}{2} \rho \beta\left(\varepsilon D_{0}\right)^{2 / 3}\right)^{2}\left[D^{* 2 / 3}-1 / W e_{7}\right]\left[\left(1-D^{* 3}\right)^{2 / 9}-1 / W e_{7}\right] .
$$

Finally, Martínez-Bazán et al. (1999b) obtained the daughter p.d.f. of $D^{*}, f^{*}\left(D^{*}\right)$. using the normalization condition $\int_{0}^{1} f^{*}\left(D^{*}\right) \mathrm{d} D^{*}=1$, as

$$
f^{*}\left(D^{*}\right)=\frac{P\left(D^{*}\right)}{\int_{0}^{1} P\left(D^{*}\right) \mathrm{d} D^{*}}=\frac{\left[D^{* 2 / 3}-A^{5 / 3}\right]\left[\left(1-D^{* 3}\right)^{2 / 9}-A^{5 / 3}\right]}{\int_{D_{\min }^{*}}^{D_{\operatorname{mix}}^{*}}\left[D^{* 2 / 3}-A^{5 / 3}\right]\left[\left(1-D^{* 3}\right)^{2 / 9}-A^{5 / 3}\right] \mathrm{d} D^{*}} .
$$

where $f^{*}\left(D^{*}\right)=D_{0} f\left(D ; D_{0}\right)$. This final normalization step is misleading and implies that the p.d.f. provided by (3.4) does not conserve volume. Thus, the probability functions given in (3.1)-(3.3) were mistakenly expressed as a function of $D^{*}$ rather than in terms of volume, $V^{*}$, and the correct way to express (3.2) should be

$$
P_{V}\left(V^{*}\right) \propto\left(\frac{1}{2} \rho \beta\left(\varepsilon D_{0}\right)^{2 / 3}\right)^{2}\left[V^{* 2 / 9}-\Lambda^{5 / 3}\right]\left[\left(1-V^{*}\right)^{2 / 9}-\Lambda^{5 / 3}\right] \text {, }
$$

where $V^{*}=V / V_{0}$ is the dimensionless volume of the product bubble. Applying now the normalization condition $\int_{0}^{1} f_{V}^{*}\left(V^{*}\right) \mathrm{d} V^{*}=1$, which at the same time conserves volume, one obtains

$$
f_{V}^{*}\left(V^{*}\right)=\frac{P_{V}\left(V^{*}\right)}{\int_{0}^{1} P_{V}\left(V^{*}\right) \mathrm{d} V^{*}}=\frac{\left[V^{* 2 / 9}-\Lambda^{5 / 3}\right]\left[\left(1-V^{*}\right)^{2 / 9}-\Lambda^{5 / 3}\right]}{\int_{V_{\max }^{*}}^{V_{\max }^{*}}\left[V^{* 2 / 9}-\Lambda^{5 / 3}\right]\left[\left(1-V^{*}\right)^{2 / 9}-\Lambda^{5 / 3}\right] \mathrm{d} V^{*}} .
$$

One can then easily transform (3.6) into a p.d.f. in terms of diameter using the condition $f_{V}^{*}\left(V^{*}\right) \mathrm{d} V^{*}=f^{*}\left(D^{*}\right) \mathrm{d} D^{*}$, with $V^{*}=D^{* 3}$ and $\mathrm{d} V^{*}=3 D^{* 2} \mathrm{~d} D^{*}$, as

$$
f^{*}\left(D^{*}\right)=3 D^{* 2} f_{V}^{*}\left(V^{*}\right) \text {. }
$$

Therefore the correct, volume conserving, expression for $f^{*}\left(D^{*}\right)$ can be given by

$$
f^{*}\left(D^{*}\right)=\frac{D^{* 2}\left[D^{* 2 / 3}-A^{5 / 3}\right]\left[\left(1-D^{* 3}\right)^{2 / 9}-A^{5 / 3}\right]}{\int_{D_{\text {min }}^{*}}^{D_{\max }^{*}} D^{* 2}\left[D^{* 2 / 3}-A^{5 / 3}\right]\left[\left(1-D^{* 3}\right)^{2 / 9}-A^{5 / 3}\right] \mathrm{d} D^{*}},
$$

which differs from the original equation (3.4) in the $D^{* 2}$ factor.

Figure 1(a) shows that the daughter-bubble p.d.f. is in fact symmetric when expressed in terms of volume as given by (3.6). On the other hand, figure 1(b) compares the daughter-bubble-size p.d.f. obtained from (3.4) and (3.8) respectively for $\varepsilon=3.5 \mathrm{~m}^{2} \mathrm{~s}^{-3}$ and $\varepsilon=10 \mathrm{~m}^{2} \mathrm{~s}^{-3}$ and a mother bubble of diameter $D_{0}=3 \mathrm{~mm}$. It is clearly shown in this figure that $f^{*}\left(D^{*}\right)$ given by (3.8) slightly differs from that given in (3.4) as $\varepsilon$ increases.

We wish to point out here that the evolutions of the bubble-size distribution presented in Martinez-Bazán et al. (1999b) were obtained by integrating the population balance equation with a Monte Carlo Method, generating a distribution of daughter bubbles using (3.2) without applying the normalization condition, and guaranteeing that volume was always conserved. Thus, the results presented in their $\$ 3.1$ are correct at the same time that they preserve volume. Furthermore, Lasheras et al. (2002) had already detected the mistake reported above, and the calculations they presented were performed using a finite differences method to solve the population balance equation with the daughter-bubble-size p.d.f. already corrected and given by 

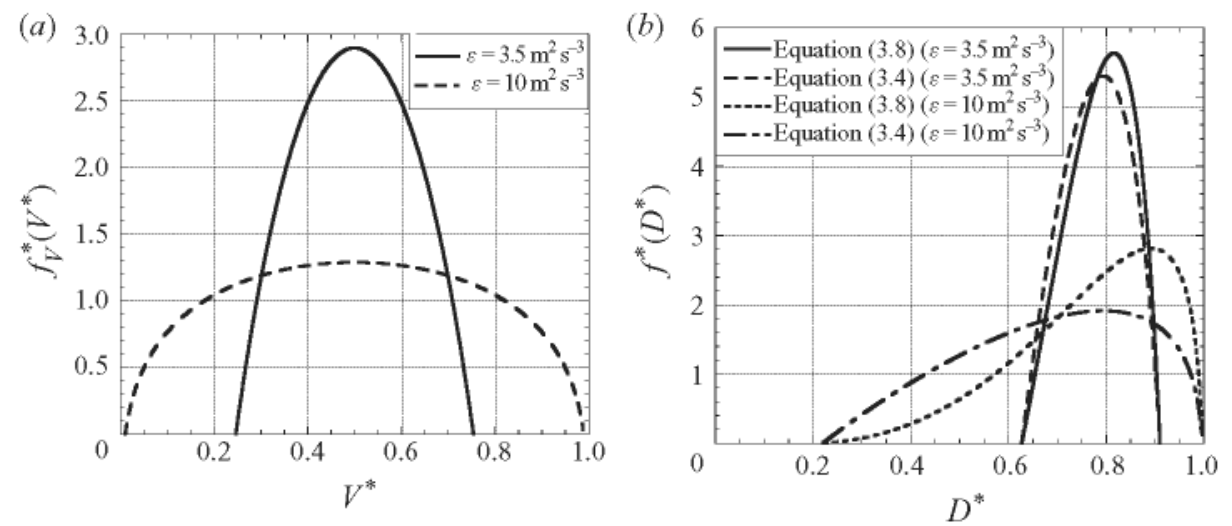

Figure 1. (a) Daughter-bubble p.d.f. as a function of the bubble volume, given by (3.6) for two different values of $\varepsilon$, showing that $f_{V}^{*}\left(V^{*}\right)$ satisfies the symmetry property. (b) Comparison of the daughter-bubble p.d.f. resulting from (3.4) and (3.8) at two different values of the dissipation rate of turbulent kinetic energy.

(3.8). As a matter of fact, mass conservation was checked in all the cases presented in the paper, obtaining that mass was preserved with errors below $0.3 \%$. In fact, as many authors do, if the population balance equation were expressed using volume as independent variable instead of diameter, the conservation equation would be automatically satisfied as (3.6) shows.

\subsection{Grant's modification of the Martinez-Bazán et al. (1999b) model}

This new model, based on the Martínez-Bazán et al. (1999b) model, includes the effect of the surface energy of the product bubbles, proposing that the probability of formation of a certain pair of bubbles should be proportional to the product of the excess stresses associated with the length scales corresponding to each bubble size, and inversely proportional to the product of surface tension energies associated with both bubbles, providing

$$
\left.\begin{array}{ll}
P_{V}\left(V^{*}\right)=0 & \text { for } \quad V^{*}<V_{\min }^{*}, \\
P_{V}\left(V^{*}\right) \propto \frac{\left[\Delta \tau_{t 1}\right]\left[\Delta \tau_{t 2}\right]}{V^{* 2 / 3}\left(1-V^{*}\right)^{2 / 3}} & \text { for } \quad V_{\min }^{*} \leqslant V^{*} \leqslant V_{\max }^{*}, \\
P_{V}\left(V^{*}\right)=0 & \text { for } \quad V^{*}>V_{\max }^{*} .
\end{array}\right\}
$$

Under these assumptions the daughter-bubble p.d.f. can be given in terms of $V^{*}$ as

$$
f_{V}^{*}\left(V^{*}\right)=\frac{V^{*-2 / 3}\left(1-V^{*}\right)^{-2 / 3}\left[V^{* 2 / 9}-\Lambda^{5 / 3}\right]\left[\left(1-V^{*}\right)^{2 / 9}-\Lambda^{5 / 3}\right]}{\int_{V_{\min }^{*}}^{V_{\max }^{*}} V^{*-2 / 3}\left(1-V^{*}\right)^{-2 / 3}\left[V^{* 2 / 9}-\Lambda^{5 / 3}\right]\left[\left(1-V^{*}\right)^{2 / 9}-\Lambda^{5 / 3}\right] \mathrm{d} V^{*}} .
$$

Equation (3.10) can be expressed in terms of $D^{*}$ as

$$
f^{*}\left(D^{*}\right)=\frac{\left(1-D^{* 3}\right)^{-2 / 3}\left[D^{* 2 / 3}-\Lambda^{5 / 3}\right]\left[\left(1-D^{* 3}\right)^{2 / 9}-\Lambda^{5 / 3}\right]}{\int_{D_{\min }^{*}}^{D_{\max }^{*}}\left(1-D^{* 3}\right)^{-2 / 3}\left[D^{* 2 / 3}-\Lambda^{5 / 3}\right]\left[\left(1-D^{* 3}\right)^{2 / 9}-\Lambda^{5 / 3}\right] \mathrm{d} D^{*}},
$$

which also satisfies the symmetry relation and conserves volume.

Figure 2 displays a comparison between the daughter-bubble p.d.f. provided by equations (3.10)-(3.11) and those given by (3.6)-(3.8), for a mother bubble of diameter 

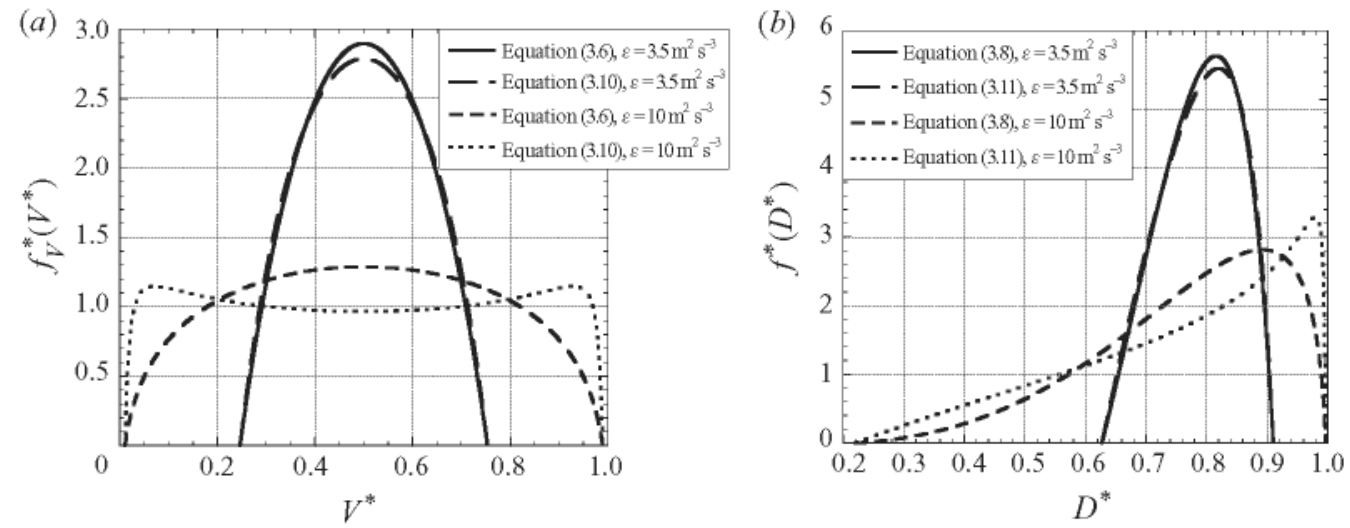

FiguRE 2. (a) Daughter-bubble p.d.f. as a function of the bubble volume, given by (3.6) and (3.10) for two different values of $\varepsilon$, showing that $f_{V}^{*}\left(V^{*}\right)$ satisfies the symmetry property. $(b)$ Comparison of the daughter-bubble p.d.f. resulting from (3.8) and (3.11) at two different values of the dissipation rate of turbulent kinetic energy.

$D_{0}=3 \mathrm{~mm}$ and two different values of the dissipation rate of turbulent kinetic energy, namely $\varepsilon=3.5 \mathrm{~m}^{2} \mathrm{~s}^{-3}$ and $\varepsilon=10 \mathrm{~m}^{2} \mathrm{~s}^{-3}$. In particular, figure $2(a)$ shows that both p.d.f.s are symmetric when expressed in terms of volume. It is clear from figure $2(b)$ that, although (3.8) and (3.11) are different, the resulting p.d.f.s are nearly identical at low values of $\varepsilon$. Only when $\varepsilon$, or equivalently the bubble Weber number, increases, do the resulting p.d.f.s begin to display some differences. In fact, contrary to the experimental observations of Rodríguez-Rodríguez, Martínez-Bazán \& Montañes (2003), the p.d.f. proposed in (3.10) evolves from a $\cap$-shape to a $\cup$-shape as $\varepsilon$ increases.

\subsection{Konno et al. (1980) model}

In their model, Konno et al. (1980) assumed that a particle is divided into $J$ units of volume $V_{e}=V_{0} / J$, such that a fragment will always be constituted by an integer number of units, $N_{k e}$, satisfying that the sum of $m$ fragments provides

$$
\sum_{i=1}^{m} N_{k e, i}=J .
$$

Furthermore, considering the physical model that a particle will break by the interaction of turbulent eddies of size smaller than the particle and, assuming that the probability of finding a fragment of a given size is proportional to the turbulent kinetic energy of an eddy of the same size, $E\left(N_{k e}\right)$, the probability of obtaining a certain distribution of fragments $N_{k e, i}$, with $i=1, \ldots, m$, after a breakage event is

$$
f^{*} \propto \prod_{i=1}^{m} E\left(N_{k e, i}\right) .
$$

To estimate the turbulent kinetic energy of an eddy of characteristic 'volume' $N_{k e} V_{e}$, Konno et al. (1980) used the Heisenberg energy spectrum,

$$
E\left(N_{k e}\right)=1.45 \varepsilon^{2 / 3}\left(\frac{\pi}{6 V_{e} N_{k e}}\right)^{-5 / 3}\left[1+10.25 V_{e}^{3}\left(\frac{\pi}{6 V_{e} N_{k e}}\right)^{4}\right]^{-4 / 3} .
$$



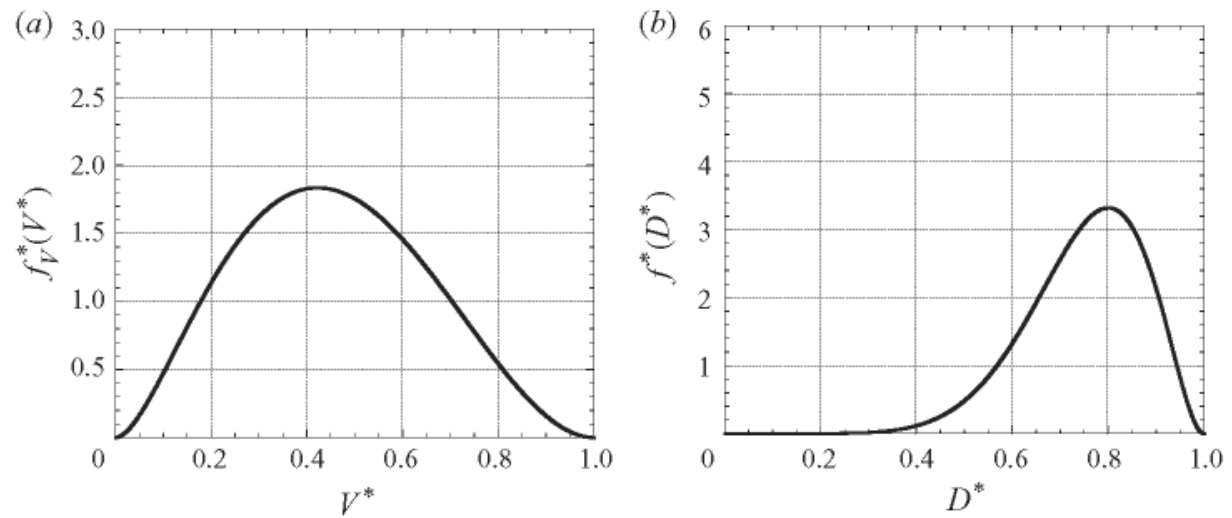

Figure 3. (a) Daughter-bubble p.d.f. as a function of the bubble volume, given by (3.16), showing that $f_{V}^{*}\left(V^{*}\right)$ does not satisfy the symmetry property since it represents a ternary breakage. (b) Daughter-bubble p.d.f. as a function of the bubble diameter, given by (3.15).

In a real system of fragments the distribution $f^{*}$ must be obtained with $J \rightarrow \infty$. However, the authors chose $J=100$ to compare with their experimental data, and obtained the best results assuming a ternary breakup where $m=3$. Finally, although the model integrates some physical ideas in the breakup process of particles, Konno, Aoki \& Saito (1983) concluded that the daughter-particle p.d.f. barely changed with the flow properties and the size of the mother particle, being in most of the cases well described by a beta function given by

$$
f^{*}\left(D^{*}\right)=\frac{\Gamma(12)}{\Gamma(3) \Gamma(9)} D^{* 8}\left(1-D^{*}\right)^{2},
$$

where $\Gamma(x)$ is the gamma function. Equation (3.15) can be expressed in terms of volume as

$$
f_{V}^{*}\left(V^{*}\right)=\frac{\Gamma(12)}{3 \Gamma(3) \Gamma(9)} V^{* 2}\left(1-V^{* 1 / 3}\right)^{2} .
$$

As can be observed in figure 3(a), the p.d.f. given by (3.15) and (3.16) does not satisfy the symmetry condition because it represents a ternary breakup process. However, since $\int_{0}^{1} D^{* 3} f^{*}\left(D^{*}\right) \mathrm{d} D^{*}=0.453 \neq 1 / 3$, it does not satisfy (2.6) and, consequently, does not preserve volume either. Unlike the experimental observations of Hesketh, Etchells \& Russell (1991), the model proposed by Konno et al. (1980, 1983) predicts that the most probable breakup event is the formation of three particles of similar sizes as shown in figure $3(b)$.

A different family of beta p.d.f.s have been proposed by Diemer \& Olson (2002) with

$$
f^{*}\left(D^{*}\right)=\frac{3 \Gamma(q m)}{\Gamma(q) \Gamma(q(m-1))} D^{* 3 q-1}\left(1-D^{* 3}\right)^{q(m-1)-1},
$$

where one of two free parameters of the beta function is the mean number of daughter fragments, $m$, and the other one, $q$, represents the shape parameter of the distribution, that must be adjusted to impose conservation of volume. For example $q=4$ in a binary breakage where $m=2, q=1.25$ if $m=3$ and $q=1.5$ if $m=4$. 


\subsection{Tsouris \& Tavlarides (1994) model}

Tsouris \& Tavlarides (1994) proposed a binary breakup model, where the probability of finding a couple of fragments of diameter $D$ and $\left(D_{0}^{3}-D^{3}\right)^{1 / 3}$, respectively, decreases as the difference between the surface energy of the mother particle and that of the daughter particles increases,

$$
\Delta E(D)=\pi \sigma D_{0}^{2}\left[D^{* 2}+\left(1-D^{* 3}\right)^{2 / 3}-1\right] .
$$

being the probability minimum if the product particles are equal, and maximum when one of the fragments has a minimum size $D_{m i n}^{*}$, defined arbitrarily in the model. Mathematically, the daughter p.d.f. was expressed as

$$
f^{*}\left(D^{*}\right)=\frac{\left(D_{\min }^{* 2}+\left(1-D_{\min }^{* 3}\right)^{2 / 3}-1\right)+2^{1 / 3}-D^{* 2}-\left(1-D^{* 3}\right)^{2 / 3}}{\int_{0}^{1}\left[\left(D_{\min }^{* 2}+\left(1-D_{\min }^{* 3}\right)^{2 / 3}-1\right)+2^{1 / 3}-D^{* 2}-\left(1-D^{* 3}\right)^{2 / 3}\right] \mathrm{d} D^{*}},
$$

an expression that does not depend on the flow conditions. However, the p.d.f. given by (3.19) does not preserve volume and does not satisfy the symmetry property. Thus, the daughter-bubble p.d.f. should have been correctly expressed in terms of volume as

$$
f_{V}^{*}\left(V^{*}\right)=\frac{\left(V_{\min }^{* 2 / 3}+\left(1-V_{\min }^{*}\right)^{2 / 3}-1\right)+2^{1 / 3}-V^{* 2 / 3}-\left(1-V^{*}\right)^{2 / 3}}{\int_{0}^{1}\left[\left(V_{\min }^{* 2 / 3}+\left(1-V_{\min }^{*}\right)^{2 / 3}-1\right)+2^{1 / 3}-V^{* 2 / 3}-\left(1-V^{*}\right)^{2 / 3}\right] \mathrm{d} V^{*}},
$$

which now satisfies the symmetry condition and at the same time that conserves volume. Equation (3.20) can be expressed in terms of diameter as

$$
f^{*}\left(D^{*}\right)=\frac{\left[\left(D_{m i n}^{* 2}+\left(1-D_{\min }^{* 3}\right)^{2 / 3}-1\right)+2^{1 / 3}-D^{* 2}-\left(1-D^{* 3}\right)^{2 / 3}\right] D^{* 2}}{\int_{0}^{1}\left[\left(D_{\min }^{* 2}+\left(1-D_{\min }^{* 3}\right)^{2 / 3}-1\right)+2^{1 / 3}-D^{* 2}-\left(1-D^{* 3}\right)^{2 / 3}\right] D^{* 2} \mathrm{~d} D^{*}} .
$$

Figure 4 shows the p.d.f. provided by (3.20) and (3.21) indicating that the distribution is symmetric. This figure also shows how the daughter p.d.f. provided by Tsouris \& Tavlarides changes as the value of $V_{m i n}^{*}$, or similarly $D_{m i n}^{*}$, is modified.

\section{Review of previous models for the breakup frequency}

A complete review of models for the breakup frequency of particles can be found in Lasheras et al. (2002) and Kolev (1993). However, in this section we will summarize some of the models with the aim of presenting them in dimensionless form.

\subsection{Martinez-Bazán et al. (1999a) model}

Martinez-Bazán et al. (1999a) established that the breakup frequency depends on the mother-bubble diameter, $D_{0}$, and on the dissipation rate of turbulent kinetic energy, $\varepsilon$, as

$$
g\left(\varepsilon, D_{0}\right)=K_{g} \frac{\sqrt{\overline{\Delta u^{2}}\left(D_{0}\right)-12 \sigma /\left(\rho D_{0}\right)}}{D_{0}}=K_{g} \frac{\sqrt{\beta\left(\varepsilon D_{0}\right)^{2 / 3}-12 \sigma /\left(\rho D_{0}\right)}}{D_{0}},
$$

where $\overline{\Delta u^{2}}\left(D_{0}\right)=\beta\left(\varepsilon D_{0}\right)^{2 / 3}$ is the mean squared value of the velocity fluctuations between two points a distance $D_{0}$ apart. Equation (4.1) can be expressed in 

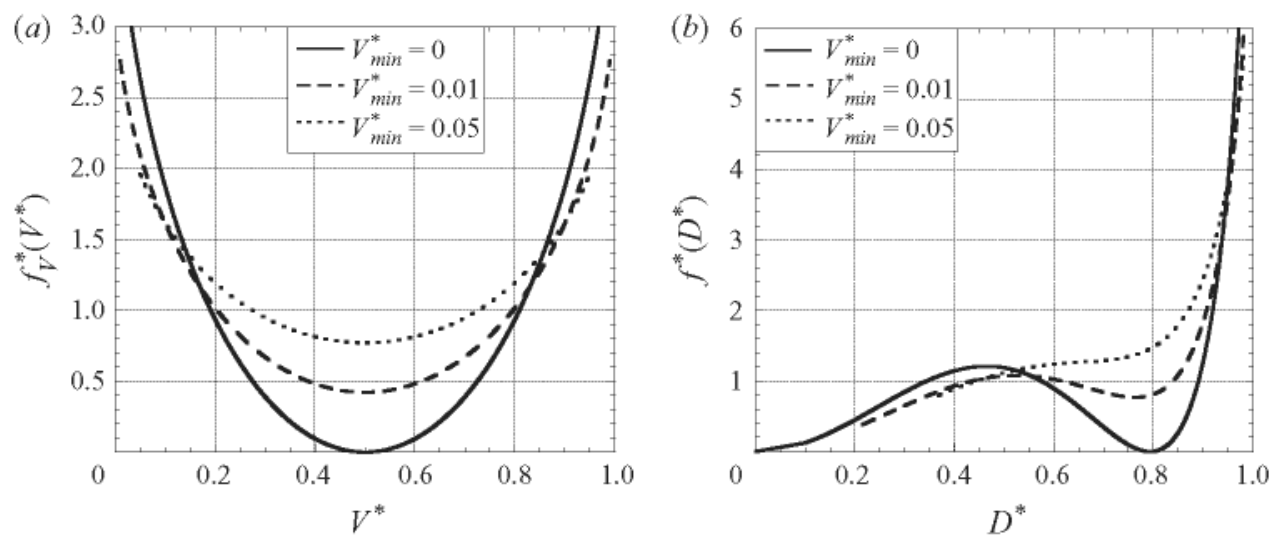

Figure 4. (a) Daughter-bubble p.d.f. as a function of the bubble volume, given by (3.20) with $V_{\min }^{*}=0,0.01$ and 0.05 respectively, showing that $f_{V}^{*}\left(V^{*}\right)$ satisfies the symmetry condition. (b) Corresponding daughter-bubble p.d.f. as a function of the bubble diameter, $f^{*}\left(D^{*}\right)$, given by $(3.21)$.

dimensionless form in terms of the turbulent Weber number, defined here as $W e_{t}=\rho \beta \varepsilon^{2 / 3} D_{0}^{5 / 3} / 12 \sigma$, as

$$
g^{*}\left(W e_{t}\right)=\frac{g\left(\varepsilon, D_{0}\right) D_{0}^{2 / 3}}{\varepsilon^{1 / 3}}=K_{g} \beta^{1 / 2} \sqrt{1-\frac{1}{W e_{t}}},
$$

where $K_{g} \beta^{1 / 2} \approx 0.673$. In general, for a different definition of the turbulent-bubble Weber number, (4.2) can be expressed as

$$
g^{*}\left(W e_{t}\right)=C_{g} \sqrt{1-\frac{W e_{t c}}{W e_{t}}},
$$

where $W e_{t c}$ is the critical Weber number and $C_{g}$ is a constant. In particular, RodríguezRodríguez, Gordillo \& Martínez-Bazán (2006) found a good agreement between the breakup time obtained from numerical simulations of the deformation process of a bubble of radius $a_{0}$ immersed in a straining flow, and that predicted by (4.3). In their case, where the dimensionless frequency was defined with the bubble radius as $g^{*}=g \varepsilon^{-1 / 3} a_{0}^{2 / 3}$ and the Weber number was $W e_{t}=3.38 \rho \varepsilon^{2 / 3} a_{0}^{5 / 3} / \sigma$, the critical Weber number was $W e_{t c}=2.3$ and $C_{g}=0.423$.

We have represented in figure 5 the evolution of the dimensionless breakup frequency with the Weber number obtained from the experiments reported by Martínez-Bazán et al. (1999a), together with the predictions of the different models considered in this work. In particular, it can be observed in this figure that the breakup model given by (4.2) reproduces correctly the experimental results, especially those of Set 1 and Set $3 b$.

\subsection{Coulaloglou \& Tavlarides (1977) model}

Coulaloglou \& Tavlarides (1977) proposed the following expression for the particle breakup frequency:

$$
g=\left(\frac{1}{\text { Breakup time }}\right) \times(\text { Fraction of particles breaking }) .
$$




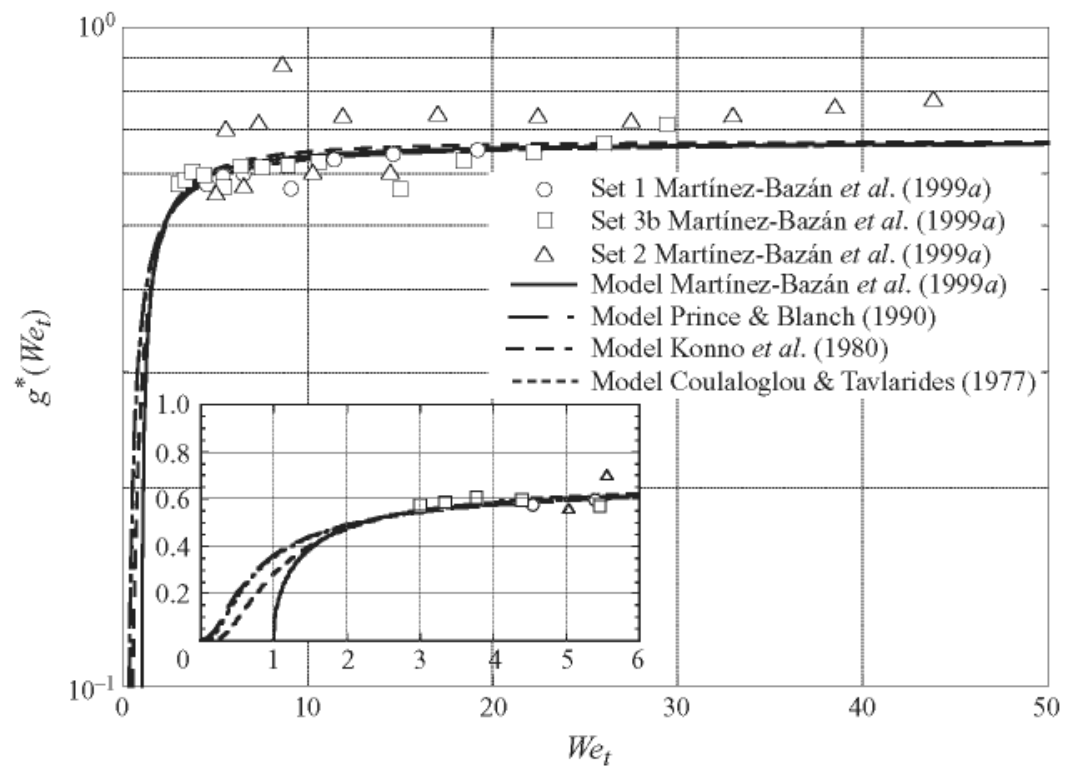

FIGURE 5. Comparison among the dimensionless breakup frequency resulting from different models (Coulaloglou \& Tavlarides 1977; Konno et al. 1980; Prince \& Blanch 1990; Martínez-Bazán et al. 1999a) and the experimental measurements of Martínez-Bazán, Montañes \& Lasheras (1999a). Here $\bigcirc, \square$ and $\triangle$ correspond respectively to the experimental data of Set 1, Set 3b and Set 2 reported in Martínez-Bazán et al. (1999a).

The authors suggested that the breakup time should scale as $t_{b} \sim \varepsilon^{-1 / 3} D_{0}^{2 / 3}$ while, based on the Boltzmann statistics, they modelled the fraction of particles breaking as

$$
\frac{\Delta n\left(D_{0}\right)}{n\left(D_{0}\right)} \propto \exp \left(-\frac{E_{a}}{\bar{E}}\right),
$$

where $E_{a} \sim \sigma D_{0}^{2}$ is the particle's surface energy and $\bar{E} \sim \rho \varepsilon^{2 / 3} D_{0}^{11 / 3}$ is the mean kinetic energy of turbulent structures of the size of the particle. Thus, the breakup frequency of a particle of size $D_{0}$ can be given by

$$
g\left(\varepsilon, D_{0}\right)=C_{c 1} D_{0}^{-2 / 3} \varepsilon^{1 / 3} \exp \left(-C_{c 2}^{\prime} \frac{\sigma}{\rho \varepsilon^{2 / 3} D_{0}^{5 / 3}}\right),
$$

where $C_{c 1}$ and $C_{c 2}^{\prime}$ are constants to be adjusted experimentally. Equation (4.6) can be expressed in dimensionless form as

$$
g^{*}\left(W e_{t}\right)=\frac{g\left(\varepsilon, D_{0}\right) D_{0}^{2 / 3}}{\varepsilon^{1 / 3}}=C_{c 1} \exp \left(-\frac{C_{c 2}}{W e_{t}}\right),
$$

with $C_{c 2}=C_{c 2}^{\prime} \beta / 12$. Notice that the breakup frequency proposed by Coulaloglou \& Tavlarides (1977) tends exponentially to an asymptotic value given by $g^{*}=C_{c 1}$ when $W e_{\mathrm{t}} \rightarrow \infty$, while it becomes exponentially small when $W e_{\mathrm{t}} \rightarrow 0$. This last result implies that this model does not involve the existence of a critical Weber number as the model proposed by Martínez-Bazán et al. (1999a).

The evolution of the dimensionless breakup frequency given by this model has also been plotted in figure 5 with the aim of comparing it with the experimental results, as well as with the predictions provided by other models. Thus, figure 5 shows that the 
breakup frequency model given by (4.7) also reproduces correctly the experimental results reported by Martinez-Bazán et al. $(1999 a)$ with $C_{c 1}=0.673$ and $C_{c 2}=0.65$.

\subsection{Konno et al. (1980) model}

Based on arguments similar to those of Coulaloglou \& Tavlarides (1977), Konno et al. (1980) proposed an alternative expression for the breakup frequency given by

$$
g\left(\varepsilon, D_{0}\right)=C_{k 1}^{\prime} \frac{\sqrt{\overline{\Delta u^{2}}\left(D_{0}\right)}}{D_{0}} \int_{u_{c}}^{\infty_{c}} P(u) \mathrm{d} u
$$

where the probability $P(u)$ is given by the Maxwell distribution

$$
P(u)=4 \pi\left(\frac{3}{2 \pi \overline{\Delta u^{2}}\left(D_{0}\right)}\right)^{3 / 2} u^{2} \exp \left(-\frac{3}{2} \frac{u^{2}}{\overline{\Delta u^{2}}\left(D_{0}\right)}\right) .
$$

In this case, $C_{k 1}^{\prime}$ is a constant and $u_{c}=C_{k 2}^{\prime}\left(\sigma / \rho D_{0}\right)^{1 / 2}$ represents the critical velocity such that turbulent structures whose characteristic velocity is smaller than $u_{c}$ are not able to split the particle. Using dimensionless variables, (4.8) can be conveniently expressed as

$$
g^{*}\left(W e_{t}\right)=\frac{g\left(\varepsilon, D_{0}\right) D_{0}^{2 / 3}}{\varepsilon^{1 / 3}}=C_{k 1} \int_{\left(C_{k 2} / W_{e}\right)^{1 / 2}}^{\infty_{i}} 3 \sqrt{\frac{6}{\pi}} x^{2} \exp \left(\frac{-3 x^{2}}{2}\right) \mathrm{d} x,
$$

where $x^{2}=u^{2}\left(D_{0}\right) / \overline{\Delta u^{2}}\left(D_{0}\right)$. Notice that the lower limit in the integral of $(4.10)$ is $\left(C_{k 2} / W e_{t}\right)^{1 / 2}$, showing its dependence with the turbulent Weber number, $W e_{t}$. The model proposed by Konno et al. (1980) has also been displayed in figure 5 together with the experimental results of Martínez-Bazán et al. (1999a), indicating the good agreement between the breakup frequency predicted by this model when $C_{k 1}=0.673$ and $C_{k 2}=0.95$ and the experiments.

\subsection{Prince \& Blanch (1990) model}

Following arguments from the kinetic theory of gases, Prince \& Blanch (1990) proposed a particle breakup frequency model where the particles split due to their collision with 'fictitious' turbulent eddies. Thus, the breakup frequency should be equal to the number of collisions per unit time, multiplied by their collision efficiency. In their model, the frequency of collisions of a certain particle with the turbulent eddies present in the flow, $\theta_{D_{0} e}$, is obtained by the product of three different terms as

$$
\theta_{D_{0 e}}=n_{e} S_{D_{0} e}\left(\overline{\Delta u^{2}}\left(D_{0}\right)+\overline{\Delta u_{e}^{2}}\right)^{1 / 2}
$$

where $n_{e}$ is the number density of eddies, $S_{D_{v e}}$ is the collision cross-sectional area and $\left(\overline{\Delta u^{2}}\left(D_{0}\right)+\overline{\Delta u_{e}^{2}}\right)^{1 / 2}$ is the particle-eddy mean relative velocity with $\overline{\Delta u_{e}^{2}}$ being the mean square random velocity of an eddy of radius $r_{e}$. To obtain the number density of eddies Prince \& Blanch (1990) used the following expression:

$$
\frac{\mathrm{d} n_{e}}{\mathrm{~d} \kappa}=0.1 \kappa^{2}
$$

which provides the number of eddies found at each wavenumber, $k$. Since the integration of (4.12) over the whole energy spectrum provides an infinite number of eddies, the authors arbitrarily limited the eddies capable of splitting a particle to those whose size is larger than $20 \%$ the diameter of the particle. Moreover, the collision 
cross-sectional area can be modelled as

$$
S_{D_{0} e}=\frac{\pi}{4}\left(D_{0} / 2+r_{e}\right)^{2},
$$

where $r_{e}=\pi / \kappa$ is the radius of an eddy of wavenumber $\kappa$. As for the mean quadratic velocities, Prince \& Blanch (1990) expressed them as $\overline{\Delta u^{2}}\left(D_{0}\right)=1.96\left(\varepsilon D_{0}\right)^{2 / 3}$ for the particle and $\overline{\Delta u_{e}^{2}}=1.96\left(\varepsilon 2 r_{e}\right)^{2 / 3}$ for turbulent eddies of radius $r_{e}$ respectively. Finally, to determine the collision efficiency, $F$, the model uses an expression similar to that considered by Coulaloglou \& Tavlarides (1977), based on the Boltzmann distribution,

$$
F=\exp \left(-\frac{u_{c i}^{2}}{\overline{\Delta u_{e}^{2}}}\right)=\exp \left(-\frac{2 \times 1.52^{2} \sigma}{1.96 \rho\left(\varepsilon 2 r_{e}\right)^{2 / 3} D_{0}}\right)
$$

with $u_{c i}=1.52\left(2 \sigma / \rho D_{0}\right)^{1 / 2}$. Therefore, the breakup rate of bubbles of size $D_{0}$ is obtained by integrating over the entire spectrum of eddies considered yielding

$$
\begin{aligned}
g\left(\varepsilon, D_{0}\right)=\frac{0.14 \pi}{16} \int_{0}^{10 \pi / D_{0}}\left(D_{0}+\right. & \left.\frac{2 \pi}{\kappa}\right)^{2}\left(D_{0}^{2 / 3}+\left(\frac{2 \pi}{\kappa}\right)^{2 / 3}\right)^{1 / 2} \\
& \times \varepsilon^{1 / 3} \exp \left[-\frac{2.36}{(2 \pi)^{2 / 3}} \frac{\sigma \kappa^{2 / 3}}{\rho D_{0} \varepsilon^{2 / 3}}\right] \kappa^{2} \mathrm{~d} \kappa .
\end{aligned}
$$

Equation (4.15) can be formulated in dimensionless form as

$g^{*}\left(W e_{f}\right)=\frac{0.14 \pi}{16} \int_{0}^{10 \pi}\left(1+\frac{2 \pi}{\kappa^{*}}\right)^{2}\left(1+\left(\frac{2 \pi}{\kappa^{*}}\right)^{2 / 3}\right)^{1 / 2} \exp \left[-\frac{2.36 \beta}{12(2 \pi)^{2 / 3}} \frac{\kappa^{* 2 / 3}}{W e_{t}}\right] \kappa^{* 2} \mathrm{~d} \kappa^{*}$

where $\kappa^{*}=\kappa D_{0}$ is the dimensionless wavenumber. This model predicts a tendency of the breakup frequency similar to those given by Coulaloglou \& Tavlarides (1977) and Konno et al. (1980). However, the values of the dimensionless frequency are not of order unity, indicating that the original constants used in the model were incorrectly chosen. Thus, the model can be rewritten as

$$
g^{*}\left(W e_{1}\right)=C_{p 1} \int_{0}^{10 \pi}\left(1+\frac{2 \pi}{\kappa^{*}}\right)^{2}\left(1+\left(\frac{2 \pi}{\kappa^{*}}\right)^{2 / 3}\right)^{1 / 2} \exp \left[-C_{p 2} \frac{\kappa^{* 2 / 3}}{W e_{t}}\right] \kappa^{* 2} \mathrm{~d} \kappa^{*},
$$

with $C_{p 1}$ and $C_{p 2}$ constants to be determined experimentally. The model proposed by Prince \& Blanch (1990) has been compared in figure 5 with the experimental measurements of the breakup frequency of bubbles, showing a good agreement when $C_{p 1}=3.1 \times 10^{-5}$ and $C_{p 2}=0.0853$. Notice in this figure that the dimensionless frequency given by Prince \& Blanch $(1990)$ is nearly identical to that provided by Coulaloglou \& Tavlarides (1977).

A comparison among the dimensionless breakup frequencies resulting from the different models described above has been shown in figure 5 . It can be observed that, although the models are based on different ideas, all of them provide nearly the same evolution of $g^{*}$ with $W e_{f}$, especially at $W e_{f}>2$. As displayed in the inset of figure 5 , they only differ at Weber numbers near the critical one determined by Martinez-Bazán et al. (1999a) since the models of Prince \& Blanch (1990), Konno et al. (1980) and Coulaloglou \& Tavlarides (1977) do not involve a critical Weber number, $W e_{t c}$, such that bubbles with $W e_{t}<W e_{i c}$ will never break. 


\section{Discussion}

In this section we present the evolution of the distribution of bubbles in a turbulent water jet obtained using the different models for the daughter-bubble p.d.f. described above, and compare them with the experimental results of bubbles injected at the central axis of a turbulent water jet of Martínez-Bazán et al. (1999b). The numerical method used to integrate the population balance equation is described in $\$ 5.1$, and the comparison of the evolution of the bubble-size distribution obtained with the different models in $\$ 5.2$.

\subsection{Description of the numerical algorithm}

In the steady, quasi-one-dimensional flow, given by a turbulent water jet, since the bubbles are injected at the central axis of the water jet and radial dispersion is negligible (see figure 6 in Martínez-Bazán et al. 1999a, and information therein for additional details), the population balance equation (2.3) can be expressed as

$$
U(x) \frac{\mathrm{d}[n(D)]}{\mathrm{d} x}=\int_{D}^{\infty} m\left(D_{0}\right) f\left(D ; D_{0}\right) g\left(D_{0}\right) n\left(D_{0}\right) \mathrm{d} D_{0}-g(D) n(D),
$$

where we have considered that the bubbles are convected with the mean centreline velocity of the water jet, $U(x)$, and the other variables are as defined in $\$ 2$. In order to compute the time, or equivalently, since $\mathrm{d}() / \mathrm{d} t=U(x) \mathrm{d}() / \mathrm{d} x$, the downstream evolution, of the number density of bubbles, equation (5.1) was integrated numerically. The dependence on the bubble size was discretized by dividing the whole bubble-size range, $0<D<3 \mathrm{~mm}$, into $N=1000$ equally spaced bins. Using the resulting vector of discrete sizes, the integral in (5.1) was evaluated with the method of trapezoids. The same method was employed to compute the integral in the denominator of the daughter size p.d.f. to normalize this function (see (2.7)).

Finally, the time marching was implemented using a second-order Adams-Moulton method. An implicit method was chosen to avoid numerical instabilities observed when integrating the population balance equation with some of the breakup models considered in this paper, especially when a large number of size bins was used. The numerical results shown in figures 6 and 7 were obtained with a time step $\mathrm{d} t=10^{-5} \mathrm{~s}$. A sensitivity analysis of both the time step and the number of size bins was performed to ensure that the numerical results did not depend on these parameters.

It is important to point out that the numerical method described above does not guarantee the volume-conservation condition (2.6) and, thus, it can be observed how the models that mathematically conserve volume exhibit small changes in the total bubble volume along the integration time, due to numerical errors (figure $7 b$ ). On the contrary, models with daughter-bubble-size p.d.f.s which do not conserve volume predict changes in the total volume of order unity, as can be clearly seen in figure $7(a)$.

\subsection{Comparison of the evolution of the bubble-size distribution obtained with different models}

To integrate (5.1), in the present work we always used the bubble breakup frequency model proposed by Martínez-Bazan et al. (1999a) and varied the model for the daughter-bubble p.d.f. with the aim of describing the main differences among the models. Notice that, in figure 5 all the breakup models provide nearly the same values of the dimensionless breakup frequency, showing only slight differences at low Weber numbers, $W e_{r}<2$. 
(a)

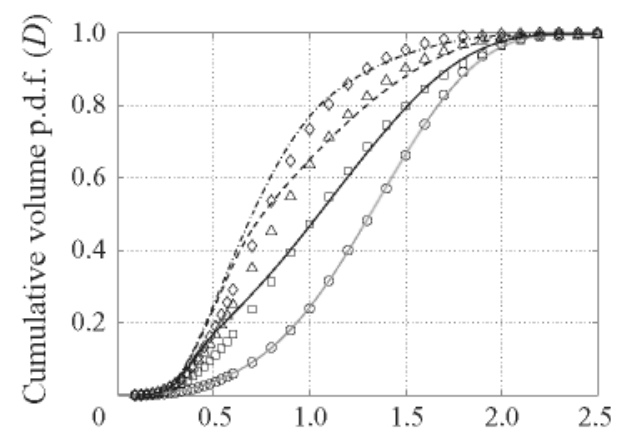

(c)

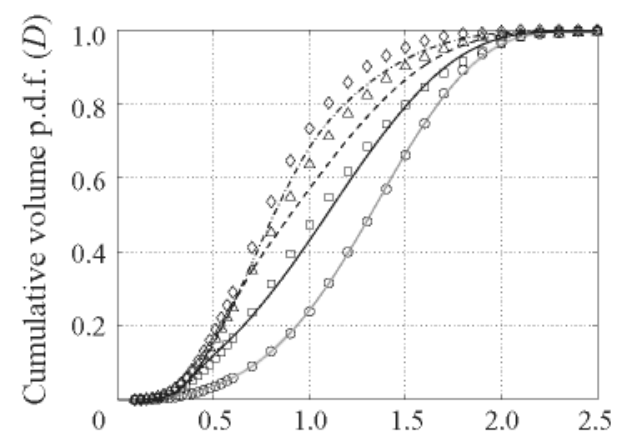

(e)

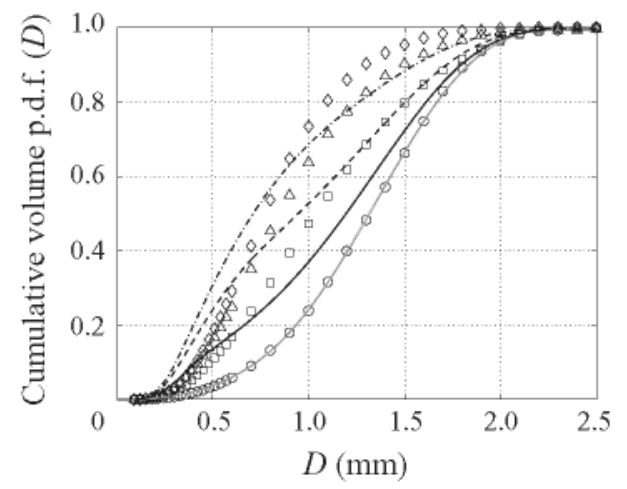

(b)

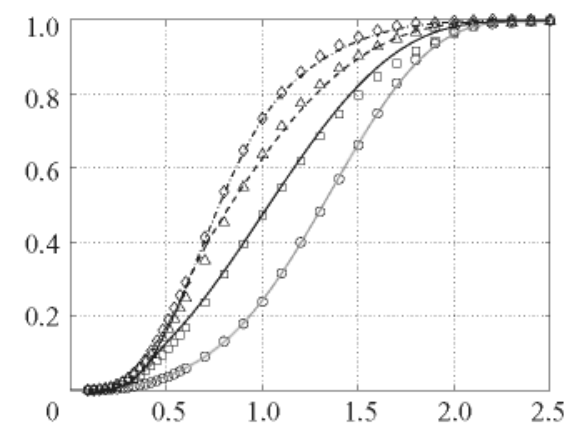

(d)

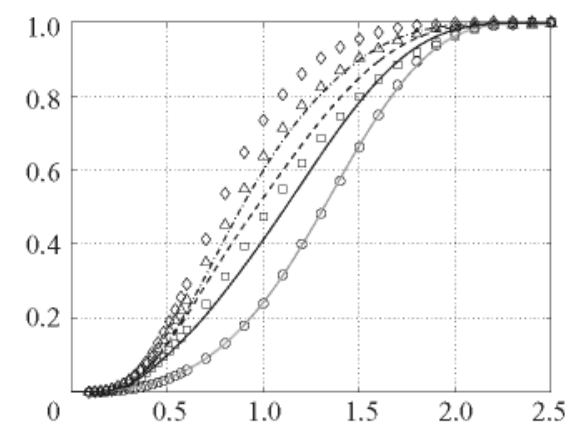

$(f)$

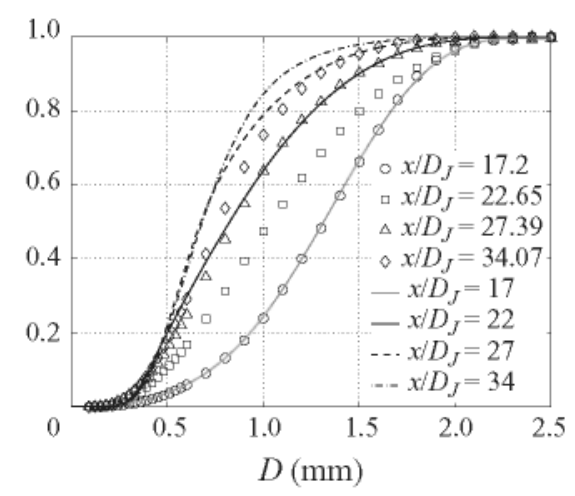

FIGURE 6. Downstream evolution of the cumulative volume p.d.f. for air bubbles injected at the centreline of a turbulent water jet obtained with different models proposed for the daughter-bubble p.d.f.: (a) Martínez-Bazán, Montañes \& Lasheras (1999b) model given by (3.4), (b) volume-conserving Martínez-Bazán et al. (1999b) model given by (3.8), (c) modified Martínez-Bazán et al. (1999b) model given by (3.11), (d) volume-conserving Tsouris \& Tavlarides (1994) model given by (3.21), (e) Tsouris \& Tavlarides (1994) model given by (3.19) and $(f)$ Konno et al. (1983) model given by (3.15). The population balance equation (5.1) was integrating using in all cases the breakup frequency model proposed by Martínez-Bazán et al. (1999a), given by (4.2) with $K_{8} \beta^{1 / 2} \approx 0.673$. The symbols represent the experimental data reported in Set 3a of Martínez-Bazán et al. (1999b).

Figure 6 shows a comparison of the downstream evolution of the cumulative volume p.d.f. of bubbles injected at the centreline of a turbulent water jet of Reynolds number $R e=25500$, obtained with the models described in $\S 3$ for the daughter-bubble 

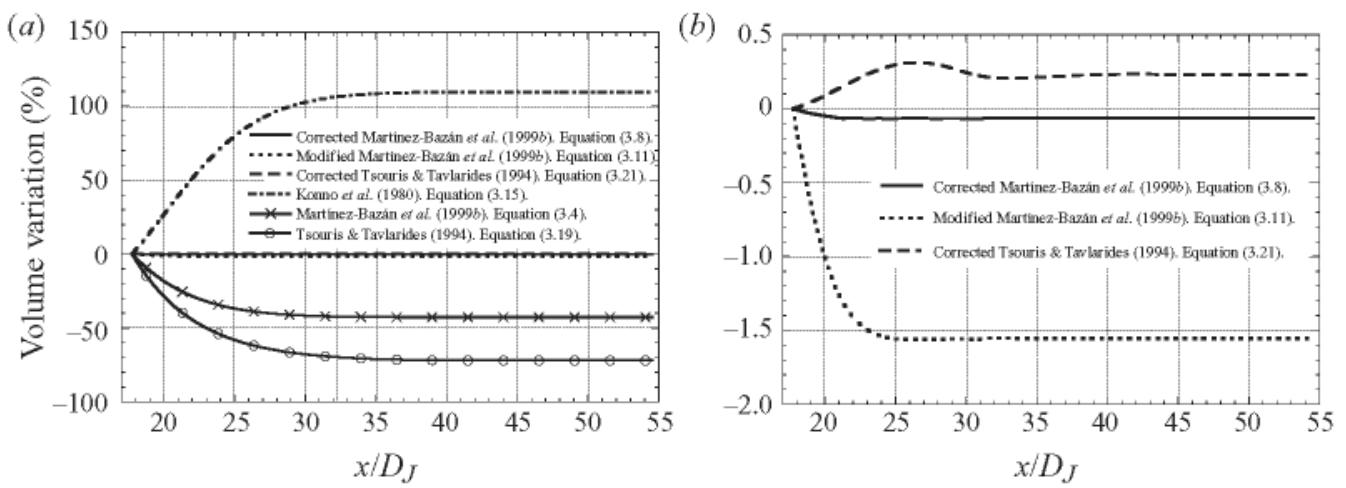

FIGURE 7. (a) Evolution of the conservation of volume obtained with our numerical code using different models proposed for the daughter-bubble p.d.f. $(b)$ Detail of the evolution of the conservation of volume obtained using some of the volume-conserving models.

p.d.f. The experimental conditions correspond to those of Set 3a of MartínezBazán et al. (1999b). Air bubbles were injected 15 diameters downstream from the water nozzle, $x / D_{J}=15$, with a hypodermic needle of inner diameter $0.394 \mathrm{~mm}$. The initial condition used to integrate the population balance equation (5.1) was the bubble-size distribution existing at $x / D_{J}=17.2$ and the water velocity and the dissipation rate of turbulent kinetic energy varied from $U=5.17 \mathrm{~m} \mathrm{~s}^{-1}$ and $\varepsilon=$ $741 \mathrm{~m}^{2} \mathrm{~s}^{-3}$ at $x / D_{J}=17$ to $U=2.50 \mathrm{~m} \mathrm{~s}^{-1}$ and $\varepsilon=22 \mathrm{~m}^{2} \mathrm{~s}^{-3}$ at $x / D_{J}=35$. The dissipation rate of turbulent kinetic energy, $\varepsilon$, was obtained by integrating the onedimensional spectrum of the fluctuating component of the axial velocity measured with hot-film anemometry, assuming the turbulence to be locally homogeneous and isotropic. In addition, coalescence effects were negligible in the experiments since the bubble void fraction was always smaller than $10^{-5}$ (more details about the experimental characterization of the turbulent properties of the water jet and the measurements of the bubble-size distribution can be found in Martínez-Bazán et al. $1999 a, b)$. Figure 6(a) shows that the non-conservative model proposed by MartínezBazán et al. (1999b) and given by (3.4) does not describe correctly the evolution of the cumulative bubble-volume p.d.f. Although the model captures the formation of large bubbles, it fails to reproduce the downstream evolution of bubbles of diameter smaller than $1 \mathrm{~mm}$. In addition, figure 7(a) shows that the implementation of the MartínezBazán (1999b) model as in (3.4) leads to a loss of volume of nearly $50 \%$. However, notice in figure $6(b)$ that, when this model is correctly implemented as in (3.8), the agreement with the experimental results is excellent with errors in the conservation of volume lower than $0.1 \%$ (see figure $7 b$ ). Similar results have been found using the modified Martínez-Bazán (1999b) model given by (3.11) which includes the effect of the surface energy of the product bubbles as shown in figure 6(c). This modified model seems to slightly underpredict the downstream evolution of the population of bubbles, especially at $x / D_{J}=27$, but the overall agreement with the experimental measurements is fairly good and, as displayed in figure $7(b)$, it conserves volume with errors smaller than $2 \%$ caused by the numerical scheme.

The results obtained from the model of Tsouris \& Tavlarides (1994) are shown in figures $6(d)$ and $6(e)$ using the volume conserving (see (3.21)) and the original (see (3.19)) expressions, respectively. Figure 7(a) shows that the original model, given by (3.19), leads to a loss of volume of the order of $50 \%$ while the volume-conserving 
model given by $(3.21)$ conserves volume with errors smaller than $0.5 \%$ as shown in figure $7(b)$. Nevertheless, this model does not adequately capture the physics of the bubble breakup process that takes places in a turbulent flow, and underpredicts the evolution of the volume p.d.f.

The downstream evolution of the bubble cloud predicted by Konno et al. (1980, 1983 ) is plotted in figure $6(f)$. As already described by Martínez-Bazán et al. (1999b) and Lasheras et al. (2002), this model overpredicts the breakup process, especially at the initial positions where the values of $\varepsilon$ are larger, since the probability of formation of bubbles smaller than $D^{*}<0.3$ is negligible. In addition, figure $7(a)$ indicates that the implementation of the model leads to a gain of volume of more than $100 \%$.

A summary of the loss (gain) of volume of the models discussed above is displayed in figure 7. Figure 7( $a$ ) shows that the Martinez-Bazán et al. (1999b) model expressed as in (3.4) and the Tsouris \& Tavlarides (1994) model expressed as in (3.19) lead to a loss of volume of larger than $45 \%$, while the Konno et al. (1983) model given by (3.15) gains over $100 \%$ of volume. However, when the models of Martinez-Bazán et al. (1999b) and Tsouris \& Tavlarides (1994) are expressed correctly as in (3.8) and (3.21) respectively, the conservation of volume property is satisfied with errors lower than $0.5 \%$, as shown in figure $7(b)$, associated with the numerical scheme used to integrate the population balance equation (5.1).

Observe that the downstream evolutions of the cumulative volume p.d.f. obtained by integrating the population balance equation (5.1) using the daughter-bubble p.d.f.s proposed by Martínez-Bazán et al. (1999b) and Tsouris \& Tavlarides (1994), shown in figures $6(b)$ and $6(d)$ respectively, agree with those reported in Martinez-Bazán et al. (1999b) as well as in Lasheras et al. (2002). This agreement indicates that, although the models were not adequately expressed, they were correctly formulated and implemented in their volume-conserving form in Martinez-Bazán et al. (1999b) and Lasheras et al. (2002).

\section{Conclusions}

As with many other problems involving the dynamics of a population of particles, the evolution of the size distribution of a population of bubbles immersed in a fully developed turbulent flow can be modelled using the population balance equation. In this process, when the bubble concentration, or void fraction, is dilute and neglecting gas dissolution effects, the main cause of change in the bubble distribution can be attributed to breakup. Since this is the case in many practical applications, several researchers have become interested in the problem over the years, which has resulted in a number of models required to integrate the population balance equation.

Despite the many different physical approaches adopted by researchers while developing their models, we have shown that, when dimensional analysis is applied, most of the breakup frequency models predict very similar functions. In fact, we have shown that when the breakup frequency is made dimensionless with the inverse of the characteristic turnover time of turbulent eddies of sizes comparable to the diameter of the bubble, all the models seem to reach an asymptote at relatively low values of a properly defined turbulent Weber number, $W e_{t} \approx 5$, and the breakup frequency quickly decreases as the Weber number decreases. Since this is a relatively low value in most practical applications, a first conclusion is that the exact shape of the bubble breakup frequency at low Weber numbers might not be important to characterize the time evolution of the bubble population in turbulent flows. 
In the case of the daughter-bubble-size p.d.f., there are mainly two kinds of shapes given by the different models: U-shaped curves that predict a low probability of formation of fragments of comparable sizes and that could be justified by the fact that symmetric binary breakups require more surface energy (Wang, Wang \& Jin 2003), and $n$-shaped curves that predict a high probability of formation of bubbles of nearly the same size. Thus, we have performed numerical computations using different models while keeping the same breakup frequency function, with the purpose of evaluating the effect of the shape of the daughter-bubble-size p.d.f. on the time evolution of a population of bubbles. Our results indicate that binary models with n-shaped daughter-bubble-size p.d.f.s achieved a better agreement with experimental measurements. This result agrees with those reported by Zaccone et al. (2007) which. based on energy considerations, justified the presence of a maximum probability of formation of particles of similar size, and indicated that the change in surface energy is negligible compared with other type of energies involved in a breakup event.

Despite the precise shape of the daughter size p.d.f., any model must satisfy the volume-conservation condition and normalization conditions given by (2.6) and (2.7), respectively. Remarkably, some of the models proposed in the literature do not satisfy the volume-conservation condition when they are expressed in terms of the bubble diameter instead of its volume. Nevertheless, when the change of variable from volume to diameter is properly performed for a probability density, the conservation of volume condition is satisfied. To see the effect of using a p.d.f. not properly transformed, numerical computations have been also performed using a numerical method that do not enforce volume conservation. Besides the obvious errors in total bubble volume, it can be concluded that the p.d.f.s not properly transformed tend to predict a large number of very small bubbles that are not observed in experiments.

This research was supported by the Spanish MICINN under Projects\# DPI200806624-C03-02 and DPI2008-06369. Part of this work has been extracted from the PhD thesis of Javier Rodriguez-Rodriguez (Rodriguez-Rodriguez 2004).

\section{REFERENCES}

Coulaloglou, C. A. \& Tavlarides, L. L. 1977 Description of interaction processes in agitated liquid-liquid dispersions. Chem. Engng Sci. 32, 1289-1297.

Deane. G. B. \& Stokes. M. D. 2002 Scale dependence of bubble creation mechanisms in breaking waves. Nature 418, 839-844.

Difmer, R. B. \& Olson, J. H. 2002 A moment methodology for coagulation and breakage problems. Part 3. Generalized daughter distribution functions. Chem. Engng Sci. 57, 4187-4198.

Hesketh, R. P.. Etchells, A. W. \& Russell, T. W. F. 1991 Bubble breakage in pipeline flow. Chem. Enging Sci. 46, 1-9.

HINZE J. O. 1955 Fundamentals of the hydrodynamics mechanisms of splitting in dispersion process. AIChE J. 1, 289-295.

Kolev, N. I. 1993 Fragmentation and coalescence dynamics in multiphase flows. Exp. Therm. Fluid Sci. 6, 211-251.

Kolmogorov, A. N. 1949 On the breakage of drops in a turbulent flow. Dokt. Akad. Navk. SSSR 66, 825-828.

KonNo, M., AOKI, M. \& SAITO, S. 1983 Scale effect on breakup process in liquid-liquid agitated tanks. J. Chem. Engng Japan 16, 312-319.

Konno, M.. Matsunaga, Y.. Arai. K. \& Saito, S. 1980 Simulation model for breakup process in an agitated tank. J. Chem. Engng Japan 13, 67-73.

Lasheras, J. C., Eastwood, C., Martínez-Bazán, C. \& Montañes, J. L. 2002 A review of statistical models for the break-up of an immiscible fluid immersed into a fully developed turbulent flow. Intl J. Multiph. Flow 28, 247-278. 
Luo, H. \& Svensen, H. F. 1996 Theoretical model for drop and bubble breakup in turbulent dispersions. AIChE J. 42, 1225-1233.

Martínez-Bazán, C.. Montañes, J. L. \& Lasheras, J. C. $1999 a$ On the breakup of an air bubble injected into a fully developed turbulent flow. Part 1 . Breakup frequency. J. Fluid Mech. 401, $157-182$.

Martínez-Bazán, C. Montañes, J. L. \& Lasheras, J. C. 1999 on the breakup of an air bubble injected into a fully developed turbulent flow. Part 2. Size PDF of the resulting danghter bubbles. J. Fluid Mech. 401, 183-207.

Melville, W. K. 1996 The role of surface-wave breaking in air-sea interaction. Annu. Rev. Fluid Mech. 18, 279-321.

Prince, M. J. \& BLANCH, H. W. 1990 Bubble coalescence and break-up in air-sparged bubble columns. AIChE J. 36, 1485-1499.

Rodríguez-Rodríguez, J. 2004 Estudio de la rotura de gotas y burbujas en flujos a altos números de Reynolds. PhD thesis, Universidad Carlos III de Madrid, Madrid, Spain.

Rodríguez-Rodríguez, J., Gordillo, J. M. \& Martínez-Bazán, C. 2006 Breakup time and morphology of drops and bubbles in a high-Reynolds-number flow. J. Fluid Mech. $\mathbf{5 4 8}$, $69-86$.

Rodríguez-Rodríguez, J., Martínez-Bazán, C. \& Montañes, J. L. 2003 A novel particle tracking and break-up detection algorithm: application to the turbulent break-up of bubbles. Meas. Sci. Technol. 14, 1328-1340.

Tsouris, C. \& Tavlarides, L. L. 1994 Breakage and coalescence models for drops in turbulent dispersions. AIChE J. 40, 395-406.

WANG, T. F., WANG, J. F. \& JIN, Y. 2003 A novel theoretical breakup kernel function for bubbles/droplets in turbulent flows. Chem. Engng Sci. 58, 4629-4637.

Williams, F. A. 1985 Combustion Theory, 2nd edn. Addison-Wesley.

Zaccone, A., Gäbler. A.. MaAb, S., Marchisio, D. \& Kraume, M. 2007 Drop breakage in liquid-liquid stirred dispersions: modelling of single drop breakage. Chem. Engng Sci. 62 , $6297-6307$. 